\title{
Freizeit in der Raumplanung am Beispiel einer Untersuchung über das Freizeitverhalten der Stadtberner Bevölkerung
}

\section{Einleitung}

Wir alle beanspruchen Freizeit; sie gehört zu einer der sogenannten Daseins-Grundfunktionen (VOLKART 1979). Wo sie nicht durch gewisse Alltagspflichten eingeschränkt wird, versuchen wir sie nach unseren Vorstellungen $\mathrm{zu}$ gestalten. In der Freizeit wollen wir uns vom Streß und den Zwängen des Alltagslebens erholen. Dabei können und müssen wir bei der Gestaltung der Freizeit zwischen verschiedenen Möglichkeiten abwägen. Beispielsweise werden wir eingeschränkt durch familiäre Bindungen, finanzielle Voraussetzungen oder die Ansprüche Dritter. Innerhalb solcher Randbedingungen können wir die Freizeittätigkeit, den Personenkreis, Zeit und Ort auswählen.

Freizeitaktivitäten außerhalb des eigenen Heims können.grob in freiräumliche (z. B. wandern) und anlage- resp. hausgebundene (z. B. schwimmen oder Restaurantbesuch) Aktivitäten aufgeteilt werden. Ausgehend von der Definition "Tourismus» (vgl. z. B. BERATENDE KOMMISSION FÜR FREMDENVERKEHR 1979, S. 18), sind Ausflüge in die nähere und weitere Umgebung des Wohnortes als eine besondere Form des Tourismus anzusehen. Man spricht von Eintags-, Wochenend- oder Ausflugstourismus. Für die meisten unserer Freizeittätigkeiten sind wir ebenso auf Infrastruktureinrichtungen (StraBenzufahrten, öffentliches Verkehrsnetz, Wanderwege, Lagerplätze, Parkplätze, Skilifte, Berghütten, Gaststätten, Langlauf-Loipen, Bootsstege, Badeeinrichtungen usw.) angewiesen wie auch beispielsweise auf einen frei verfügbaren Uferstreifen. Viele unserer Freizeittätigkeiten sind also mit Ansprüchen an den Raum verbunden. Im Bereich der außerstädtischen Freizeitgestaltung, auf die wir uns hier beschränken wollen, übernimmt die Landschaft die Rolle des Komplementärraumes.

Vielerorts hat der Tourismus (dazu gehört ebenso der Ferientourismus wie auch der Eintages- und Wochenendtourismus) Wohlstand gebracht, Abwanderungen gestoppt und den angestrebten regionalwirtschaftlichen Ausgleich gefördert (vgl. ELSASSER et al. 1982). Der infrastrukturintensive Ausbau der Freizeitstätten in Tourismusgebieten hat aber nicht nur zur Verbesserung der wirtschaftlichen Situation der Berggebiete beigetragen, sondern er ist auch mit verschiedenen Folgeproblemen behaftet. Bereits 1974 hat KRIPPENDORF (1975) mit seinem Buch «Die Landschaftsfres- ser» viele von uns sensibilisiert und auf die Tourismus-Interessenkonflikte hingewiesen. Neben den Ansprüchen des Touristen bestehen auch ebenso wichtige andere Ansprüche an den Raum, wie z. B.: die Ansprüche der einheimischen Bevölkerung in der Land- und Forstwirtschaft, des Natur- und Heimatschutzes und der Landesverteidigung oder Bedürfnisse der Energiewirtschaft, der Versorgung und der Reservehaltung. All diese Ansprüche nehmen laufend zu. Die verfügbare Fläche wird demzufolge immer kleiner, dies führt zu Interessenkonflikten.

\section{Die Rolle der Raumplanung}

In der Raumplanung geht es im wesentlichen darum, neue Gebietsansprüche an den Raum mit den bestehenden Strukturen - möglichst unter Vermeidung von Konflikten - zu verbinden. Ihr kommt daher im Rahmen einer gesamtheitlichen Freizeit- und Tourismuspolitik als Steuerungsinstrument zur Durchsetzung dieser räumlichen Interessen eine besondere Rolle zu. Dazu braucht es im wesentlichen Vorstellungen über gewünschte und unerwünschte Entwicklungen, eine Bedarfs- und Bestandesanalyse, eine räumliche Zuordnung nach objektiven Kriterien, den politischen Entscheidfindungsprozess und, wenn nötig, die rechtliche Festlegung (Instrument: Nutzungsplanung).

Da im Tourismus Ursache (Quellgebiet) und Wirkung (überlastete Erholungsgebiete) räumlich getrennt sind, müssen die Konflikte zwischen verschiedenartigen Interessen, wie betriebswirtschaftliche Vorteile, Schutz einzigartiger Lebensstätten oder die Ansprüche der Erholungsuchenden, nicht nur auf der lokalen Ebene ausgetragen werden. Darum ist auch eine überörtliche planerische Betrachtungsweise und Problemlösung notwendig, und darauf sind die lokalen Lösungen und Maßnahmen abzustimmen (horizontale Koordination).

In unserem föderalistischen Staatssystem fordert die Raumplanung auch ein großes $\mathrm{Maß}$ an Bereitschaft zur Koordination der raumrelevanten Aufgaben

Dr. Gilbert Thélin, wissenschaftlicher Mitarbeiter beim Bundesamt für Raumplanung, 3003 Bern 
unterschiedlicher politischer Ebenen (vertikale Koordination).

Wichtigen Bezugsrahmen für diese räumlichen $\mathrm{Ab}$ stimmungs- und Koordinationsaufgaben bildet das Bundesgesetz über die Raumplanung (RPG). Demzufolge bestimmt es die Koordination zu einer seiner Hauptaufgaben. Sie ist die Aufgabe der kantonalen Planung (Instrument: Kantonaler Richtplan). Dazu legt das Bundesgesetz die Ziele fest, und alle Planungsträger sind aufgefordert, seit 1. Januar 1980 ihre Arbeit auf diese Ziele auszurichten (Planungspflicht).

Mit Hilfe von Zielen und Grundsätzen wird der Rahmen abgesteckt, in welchem von den Planungsträgern (Bund, Kanton, Region und Gemeinde) geplant werden soll. Einzelne dieser Zielsetzungen sind auch für den Problemkreis «Freizeit - Tourismus» gültig; so werden in Art. 1 RPG der Schutz der natürlichen Lebensgrundlagen, wie Boden, Luft, Wasser, Wald, und die Landschaft, und die Erhaltung und Schaffung wohnlicher Siedlungen an erster Stelle genannt. Die Planungsgrundsätze (Art. 3 RPG) legen die Wertungen, nach welchen geplant werden soll, fest und geben Anweisungen zur Entscheidfindung. Im Zusammenhang mit der Freizeit- und Tourismusplanung sind speziell die Grundsätze zur Schonung der Landschaft und Gestaltung der Siedlungen (Art. 3 Abs. 2 u. 3 RPG) hervorzuheben: So sollen See- und Flußufer freigehalten werden, naturnahe Landschaften und Erholungsräume erhalten bleiben, Rad- und Fußwege erhalten oder geschaffen und die Siedlungen mit Grünflächen und Bäumen ausgestattet werden. In diesen Rahmen gehört auch der Grundsatz, wonach die regionalen Bedürfnisse berücksichtigt (Art.3 Abs. 4 lit. a RPG) werden müssen und Freizeitanlagen gut erreichbar sein sollen (Art. 3 Abs. 4 lit. b RPG).

\section{Offene Fragen}

Der Entscheidungsmechanismus im räumlich und strukturell komplexen Bereich Tourismus fußte bisher sehr stark auf dem Prinzip des fallorientierten und damit wenig abgestimmten Einzelentscheides (z. B. Entscheide über Wohnzonen, Zufahrten oder Skilifte). In der Regel sind auch die entsprechenden Bewilligungs- und Konzessionierungsverfahren darauf ausgerichtet. Lösungen von Freizeitproblemen werden darum sehr oft lokal, punktuell und angebotsseitig angestrebt (Ausbau einzelner Infrastruktureinrichtungen).

Ausgehend von den raumplanerischen Zielsetzungen, kann festgestellt werden, daß die Berücksichtigung freizeitrelevanter Raumansprüche samt ihren Auswirkungen eine wichtige, zu koordinierende Aufgabe der Raumplanung ist oder sein müßte.

Für ortsübergreifende gesamtheitliche Lösungsansätze fehlen oft die Kenntnisse, z. B. über das Verhaltensmuster des Einzelnen innerhalb seines räumlichen Wirkungsfeldes, über die wetterbedingten
Schwankungen des Wochenendverkehrs und über die Wünsche und Vorstellungen der Betroffenen im Quellgebiet (Stadtbewohner). Noch 1982 kommt JACSMAN (1982, S.48) zum Schluß, daß im Bereich Naherholungsverkehr in der Schweiz ein Informationsnotstand besteht. Es muß im allgemeinen Interesse liegen, solche Informationen zu beschaffen und so darzustellen, daß sie bei der Interessenabwägung in gebührendem Maße berücksichtigt werden können. Denn es besteht weiterhin die Gefahr, daß die Summe der größtenteils kleinen und eher punktuellen Eingriffe in die Umwelt in einem schleichenden Prozeß unwillkürlich zur Unterlaufung raumordnungspolitischer Zielsetzungen (z. B. des RPG oder des Schweizerischen Tourismuskonzeptes) führen kann. In besonderem Maße werden davon die noch «freien Gebiete», d.h. größtenteils Erholungsgebiete, betroffen. Aus der Sicht der Freizeitplanung ist es darum wichtig, daß in der Raumplanung auch «harte Mittel» zur Durchsetzung ihrer Ziele angewendet werden, wie beispielsweise die Beibehaltung oder Schaffung von Blockaden (sCHWARZENBACH et al. 1978, S.42) im Verkehrssystem oder beim Infrastrukturangebot. Ein solches Vorgehen erfordert aber vorerst politische Entscheide, die auf einer umfassenden Freizeitpolitik aufbauen und von klaren raumordnungspolitischen Vorstellungen ausgehen.

Es war eine der Zielsetzungen einer Untersuchung im Raume Bern (THÉLIN 1983), Angaben über das Freizeitverhalten einer Stadtbevölkerung innerhalb, und besonders auch außerhalb, der Stadt und über verhaltensbestimmende Bedürfnisse zu erhalten, um Erkenntnisse über die geographische Verteilung qualitativer und quantitativer Raumansprüche $\mathrm{zu}$ gewinnen.

\section{Darstellung und Auswertung der wichtigsten Resultate für die Raumplanung}

(Erfaßt wurden mittels vier Umfragen im März, im August und im Oktober 1975 total acht Wochenenden.)

\section{Ausflugsziele}

Die Ausflugsziele der Stadtbewohner Berns sind unerwartet weit und dispers verstreut.

- Dort wo nicht Verwandtenbesuche im Vordergrund stehen, hängt die Wahl des Zieles - vor allem bei den nähergelegenen Zielgebieten - größtenteils von der guten Erreichbarkeit und der intakten Landschaft ab.

- Der Zweitwohnsitz ist für den Besitzer (Dauermieter) bestimmender Faktor für die Zielwahl.

Kommentar: Die große Streuung der Ausflugsziele, die relativ große Zersplitterung der Freizeittätigkeiten und die hohe Ausflugsintensität lassen den Schluß zu, daß mit ortsgebundenen und tätigkeitsspezifischen Infra- 


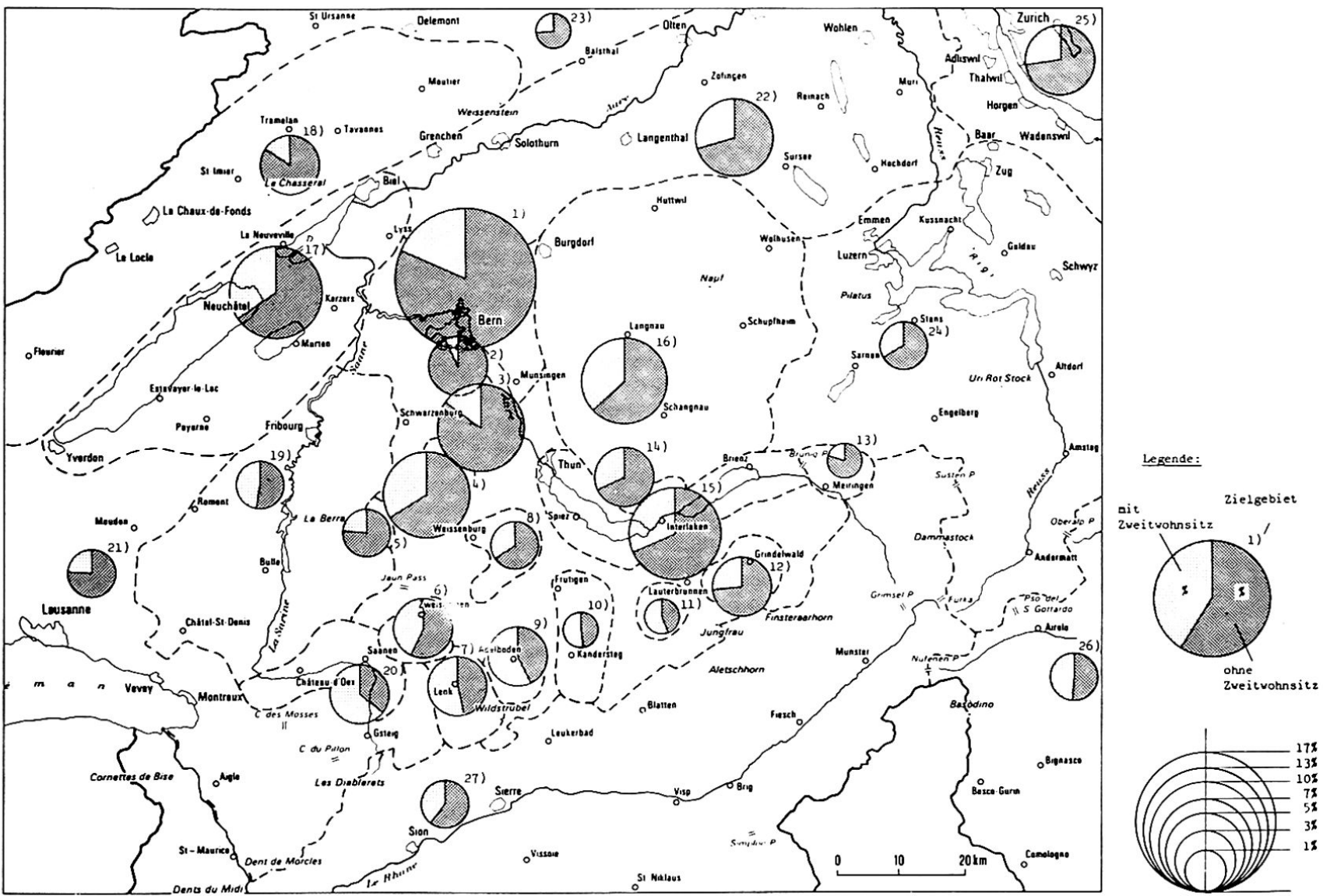

Fig. 1 Auswahl der Ausflugsgebiete im Winter, aufgeteilt nach Ausflüglern mit und ohne Zweitwohnsitz

strukturmaßnahmen - wenn überhaupt - nur in touristischen Schwerpunktsgebieten (Dreiseen-, Thunerseegebiet und gewisse Wintersportorte) mit Hilfe nutzungsplanerischer Maßnahmen Lösungen gefunden werdèn. Zudem sind bei den Verwandtenbesuchen (ca. ein Drittel der Ausflüge) und bei den Besuchen von Zweitwohnsitzen zwingende, unkorrigierbare Voraussetzungen für die Zielwahl geschaffen, die Bestrebungen für allgemeine lenkende Eingriffe zuwiderlaufen.

\section{Ausflugsmotive und Freizeitaktivitäten}

Spazieren und Wandern, Verwandtenbesuche und im Winter zusätzlich der Wintersport sind die Hauptmotive für Ausflüge. Diese Motive werden zu $60 \%$ genannt.

Durchschnittlich 9\% der Ausflügler unternehmen eine Rundfahrt.

- Der Verwandten- oder Bekanntenbesuch ist sommers und winters die beliebteste Freizeitaktivität.

Tab. 1 Ausflugsmotive in verschiedenen Untersuchungen

\begin{tabular}{|c|c|c|c|c|c|c|c|c|c|c|}
\hline & \multicolumn{2}{|c|}{$\begin{array}{l}\text { Schmidhauser } \\
1972\end{array}$} & \multirow[t]{2}{*}{\begin{tabular}{|l|} 
Albrecht \\
1965
\end{tabular}} & \multirow{2}{*}{$\begin{array}{l}\text { Friedrich et } \\
\text { al. } 1979 \\
\text { Sommer }\end{array}$} & \multicolumn{3}{|c|}{$\begin{array}{l}\text { Thélin, } \\
\text { Winter } 1975\end{array}$} & \multicolumn{3}{|c|}{$\begin{array}{l}\text { Thélin, } \\
\text { Sommer } 1975\end{array}$} \\
\hline & Winter & Sommer & & & Min. & Max. & $\varnothing$ & Min. & Max. & $\varnothing$ \\
\hline Essen/Kaffee trinken & $6 \%$ & $5 \%$ & - & $2 \%$ & $7 \%$ & $13 \%$ & $10 \%$ & $6 \%$ & $12 \%$ & $8 \%$ \\
\hline Rundfahrt & $3 \%$ & $4 \%$ & $16 \%$ & - & $6 \%$ & $12 \%$ & $9 \%$ & $6 \%$ & $11 \%$ & $9 \%$ \\
\hline $\begin{array}{l}\text { Verwandte/Bekannte } \\
\text { besuchen }\end{array}$ & $29 \%$ & $30 \%$ & - & $3 \%$ & $20 \%$ & $30 \%$ & $26 \%$ & $16 \%$ & $28 \%$ & $22 \%$ \\
\hline Spazieren/Wandern & $21 \%$ & $31 \%$ & $35 \%$ & $38 \%$ & $15 \%$ & $30 \%$ & $23 \%$ & $17 \%$ & $40 \%$ & $38 \%$ \\
\hline Wintersport & $26 \%$ & - & - & - & $9 \%$ & $26 \%$ & $19 \%$ & - & - & - \\
\hline Andere Sporttätigkeit & $1 \%$ & $3 \%$ & $15 \%$ & - & $3 \%$ & $6 \%$ & $4 \%$ & - & - & - \\
\hline Picknick & - & $8 \%$ & - & $14 \%$ & - & - & - & $1 \%$ & $4 \%$ & $3 \%$ \\
\hline Ruhen, entspannen & $4 \%$ & $5 \%$ & - & $9 \%$ & - & - & - & $13 \%$ & $18 \%$ & $17 \%$ \\
\hline Wassersport & - & $4 \%$ & $20 \%$ & - & - & - & - & $2 \%$ & $5 \%$ & $3 \%$ \\
\hline Camping & - & $2 \%$ & $3 \%$ & - & - & - & - & $1 \%$ & $6 \%$ & $3 \%$ \\
\hline Etwas anderes & $10 \%$ & $9 \%$ & $11 \%$ & $24 \%$ & $7 \%$ & $18 \%$ & $12 \%$ & $4 \%$ & $14 \%$ & $8 \%$ \\
\hline
\end{tabular}


Pro Wochenende fahren durchschnittlich $19 \%$ der Stadtberner Bevölkerung an einem oder mehreren Tagen am Wochenende zum Skilaufen.

- Die Suche nach Ruhe und Entspannung ist recht ausgeprägt und das Hauptmotiv der Zweitwohnsitzbenützer.

Kommentar: Wetterabhängige und saisonal bedingte Schwankungen sind sehr groß. Spitzentage sind daher eher selten (während der Befragungskampagne einmal auf acht Wochenenden). Unter Berücksichtigung der großen Streuung der Zielgebiete und variantenreichen Freizeitgestaltung kann angenommen werden, daß -
Zwischen 11 und $36 \%$ der Bevölkerung unternehmen Eintagesausflüge.

- 10 bis $17 \%$ der Stadtbewohner unternehmen Zweibis Viertagesausflüge.

- Die gesamte Ausflugsintensität beträgt im Winter zwischen 22 und $36 \%$ und im Sommer zwischen 25 und $50 \%$.

- Im allgemeinen ist die Reiseintensität größer, als bisher in der Schweiz angenommen.

Kommentar: Die bernischen Zahlen über die Reiseintensität zeigen, daß unter den heutigen Voraussetzungen (Arbeitszeit, Straßennetz, Wohnsituation)

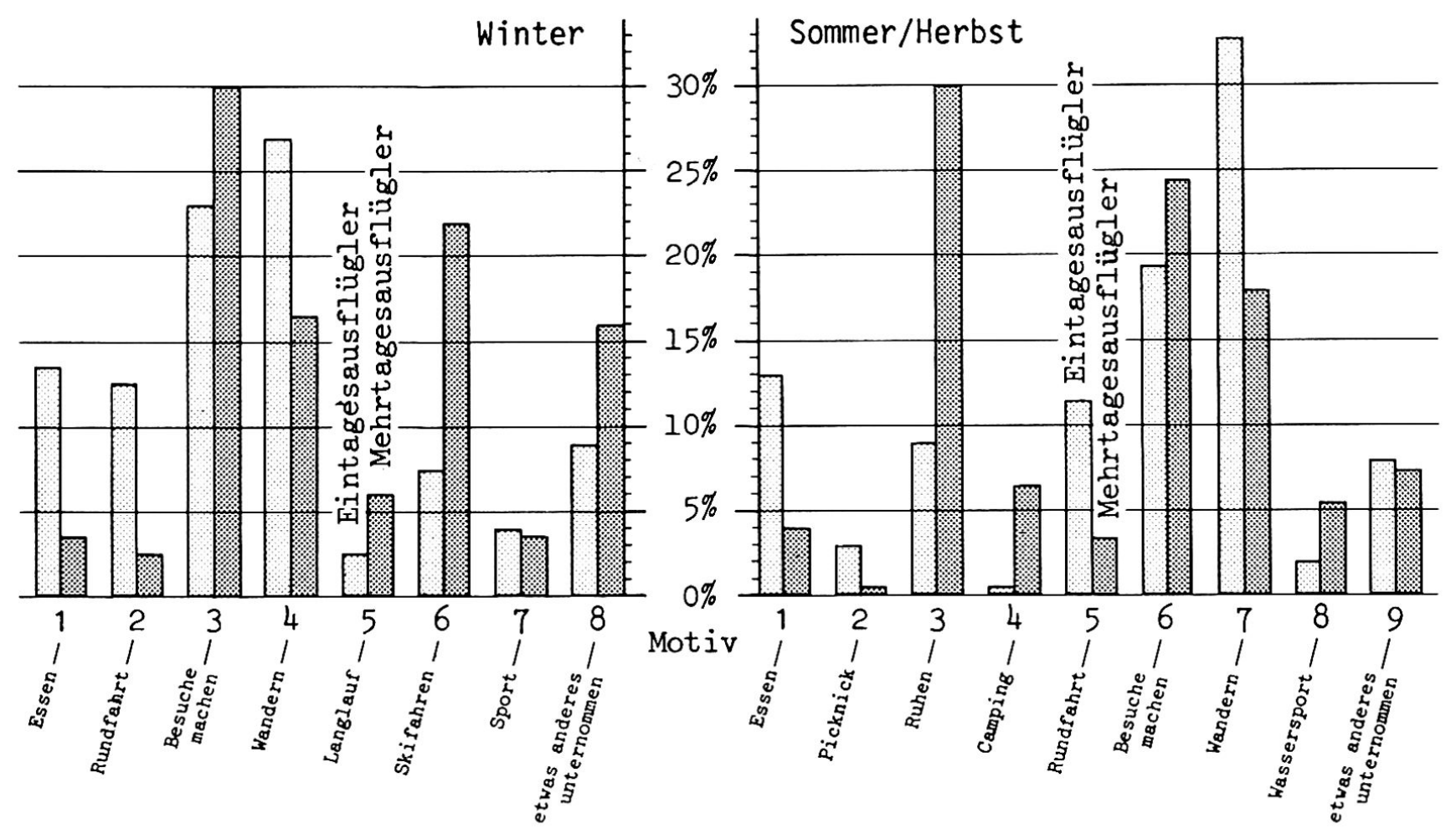

Fig. 2 Ausflugsmotive bei den Eintages- und Mehrtagesausflügen

mit Ausnahme lokaler Überbelastungen - noch ein relativ gróßes unbenutztes Potential vorhanden ist. $\mathrm{Zu}$ denken ist namentlich an Wanderwege und LanglaufLoipen. Es betrifft aber auch infrastrukturelle Einrichtungen. Die Ausnützung solcher bestehender Potentiale kann unter Umständen den schleichenden Prozeß hin zur Überentwicklung besonders wirkungsvoll unterstützen, weil gesetzliche Vorgaben für eine wirksame Kontrolle fehlen.

\section{Reiseintensität pro Wochenende}

- Eintagesausflüge sind wetterabhängig. kaum eine Steigerung der sonntäglichen Ausflugszahl zu erwarten ist. Der Druck auf Erholungsgebiete wird nur verbunden mit ganz spezifischen Aktivitäten (z. B. Langlauf, Radfahren) von Bern aus zunehmen. Schwer abzuschätzen ist der Einfluß künftiger Modetrends und die Rolle sich ändernder Wertvorstellungen.

Ein allfälliges räumliches Ausweichen ist nur noch in die heute weniger belasteten Gebiete möglich, was aber wiederum grundsätzlichen raumordnungspolitischen Zielsetzungen widerspricht und auf lange Sicht auch den Bedürfnissen der Erholungsuchenden abträglich ist (stille Erholung kaum mehr möglich). 
Tab. 2 Samstägliche und sonntägliche Reiseintensität

\begin{tabular}{|c|c|c|c|c|}
\hline & \multicolumn{2}{|c|}{ Winter } & \multicolumn{2}{|c|}{ Sommer/Herbst } \\
\hline & samstags & sonntags & samstags & sonntags \\
\hline $\begin{array}{l}\text { Eintagesausflüge } \\
\text { gewichtet }^{\star}\end{array}$ & $\begin{array}{l}17 \% \\
14 \%\end{array}$ & $\begin{array}{l}25,5 \% \\
21 \%\end{array}$ & $\begin{array}{l}19 \% \\
16 \%\end{array}$ & $\begin{array}{l}30 \% \\
24,5 \%\end{array}$ \\
\hline $\begin{array}{l}\text { Zweitagesausflüge } \\
\text { (Samstag/Sonntag) } \\
\text { gewichtet* }^{*}\end{array}$ & \multicolumn{2}{|c|}{$\begin{array}{l}4 \% \\
3,5 \%\end{array}$} & \multicolumn{2}{|c|}{$\begin{array}{l}6 \% \\
5 \%\end{array}$} \\
\hline $\begin{array}{l}\text { Mehrtagesausflüge } \\
\text { ( } 2 \text { oder } 3 \text { Übernachtungen) } \\
\text { gewichtet }^{\star}\end{array}$ & \multicolumn{2}{|c|}{$7,5 \%$} & \multicolumn{2}{|c|}{$11 \%$} \\
\hline $\begin{array}{l}\text { Total Ausflüge pro Tag } \\
\text { (Ausflugsintensität) } \\
\text { gewichtet* }\end{array}$ & $\begin{array}{l}28 \% \\
23,5 \%\end{array}$ & $\begin{array}{l}37 \% \\
30,5 \%\end{array}$ & $\begin{array}{l}37 \% \\
30,5 \%\end{array}$ & $\begin{array}{l}47 \% \\
39 \% \\
\end{array}$ \\
\hline $\begin{array}{l}\text { Total Wochenendausflüge } \\
\text { gewichtet* } \\
\text { Total Wochenendtouristen } \\
\text { gewichtet }^{\star}\end{array}$ & \multicolumn{2}{|c|}{$\begin{array}{l}53,5 \% \\
44,5 \% \\
46,5 \% \\
38,5 \%\end{array}$} & \multicolumn{2}{|c|}{$\begin{array}{l}66,5 \% \\
55 \% \\
55 \% \\
46 \%\end{array}$} \\
\hline 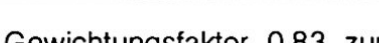 & \multicolumn{2}{|c|}{$\mathrm{n}=782$ resp. 776} & \multicolumn{2}{|c|}{$\mathrm{n}=688$ resp. 677} \\
\hline
\end{tabular}

untervertretenen Gruppe der Nichtausflügler ist bei Hochrechnungen zu berücksichtigen.

\section{Zweitwohnsitz}

- Ca. 9\% (KASPAR 1981) der Stadtberner verfügen über einen Zweitwohnsitz (Eigentum, Dauermiete), insgesamt $19 \%$ im Winter und $25 \%$ im Sommer steht ein solcher regelmäßig zur Verfügung.

- Ein Drittel (ohne Thunersee) bzw. die Hälfte aller Zweitwohnsitze befinden sich im Berner Oberland.
- Gegenüber den anderen Wochenendtouristen verbringen diejenigen, welche über einen Zweitwohnsitz verfügen, nur halb so viele Nächte im Hotel und machen nur halb so viele Gaststättenbesuche.

- Personen, die über einen Zweitwohnsitz verfügen, weisen eine erhöhte Bereitschaft für Wochenendausflüge auf; d. h. die Ausflugsintensität ist höher.

- Die durchschnittliche Reisedistanz ist größer.

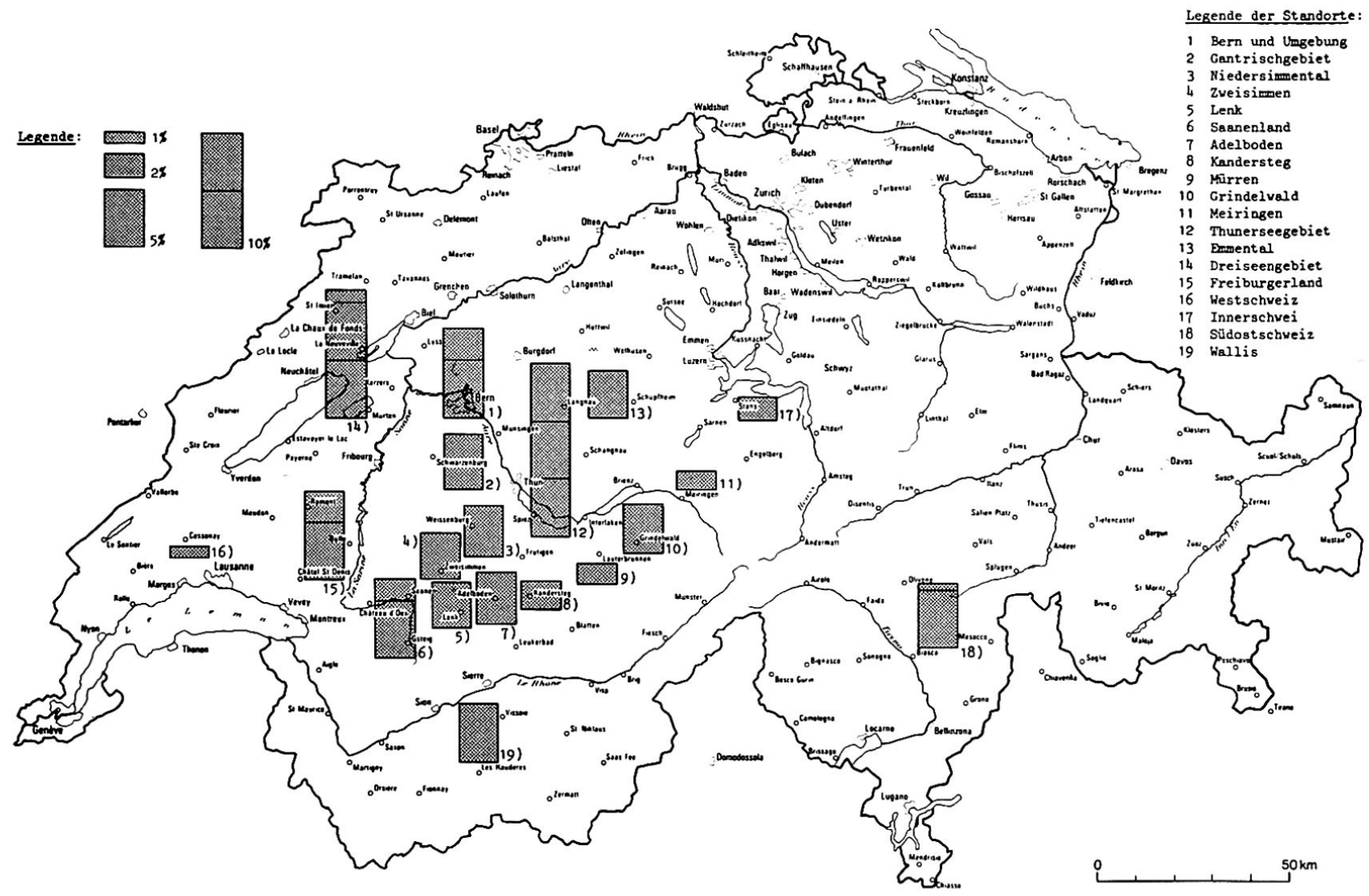

Fig. 3 Relative Verteilung der Zweitwohnsitze (Besitz, Dauermiete, regelmäßig zur Verfügung stehend) - Zusammenfassung aller vier Umfragen 
Kommentar: Der weiterhin anhaltende Zuwachs an Zweitwohnungen führt vermehrt zu Belastungserscheinungen (Landschaftsbelastung, Infrastrukturbelastung, Bodenpreissteigerung) im Zielgebiet. Die erhöhte Bereitschaft zum Wochenendausflug (erhöhte durchschnittliche Reiseintensität) ist verbunden mit einem in der Regel durchschnittlich größeren Energiekonsum, was wiederum eine größere Umweltbelastung zur Folge hat und auf Kosten anderer Erholungsuchender geht. Der Beweggrund für diese Ausflüge kann nicht auf die allgemein vertretene Fluchtthese zurückgeführt werden, wonach unbefriedigende Wohn- und Umweltbedingungen in der Großstadt den Massenexodus am Wochenende nachhaltig beeinflussen. Die Ergebnisse weisen darauf hin, daß z. B. besonders Bewohner von Einfamilienhäusern zu den Besitzern von Zweitwòhnsitzen gehören. So stellt sich denn die Frage, welche Umstände maßgebend sind für den Kauf; ist es die Kapitalinvestition, die Altersvorsorge (Alterswohnsitz), das Prestigedenken?

Die Motivation, dieser auch von politischen Kreisen und immer mehr auch Kreisen des Tourismus nicht geschätzten Entwicklung (Baustopp in Kurorten) in irgendeiner Form Einhalt zu gebieten, ist noch zu wenig groß, obschon eine eigengenutzte Zweitwoh-
Zahl Erholungsuchender, deren "Tätigkeit» und die geographische Verteilung der Zielgebiete. Im wesentlichen unterscheidet er vier Kategorien mit unterschiedlichen Raumansprüchen:

Typ 1: Erholungsuchende mit der Präferenz «klettern, wandern, ruhen, Naturbeobachtung, Langlauf usw.», Flächenbedarf: 1 ha/Person

Typ 2: Erholungsuchende mit der Präferenz «spazieren, wandern, ruhen, lagern, Radwandern, Camping, Freizeitwohnen", Flächenbedarf 1/7 ha/P.

Typ 3: Erholungsuchende mit der Präferenz «Besichtigung, promenieren, spazieren, Mannschaftssport, Tennis, schwimmen usw.», Flächenbedarf: $1 / 100 \mathrm{ha} / \mathrm{P}$.

Typ 4: Erholungsuchende mit der Präferenz «Motorrad- und Autofahren, Restaurantbesuche», Flächenbedarf: keiner.

Ausflügler, welche die Region verlassen oder zusätzlich von außen aufsuchen, werden mittels Korrekturfaktors berücksichtigt.

Die nachfolgende Berechnung geht von der Annahme aus, daß das Freizeitverhalten der Ausflügler im Kanton Bern demjenigen der Stadt Bern entspricht.

Tab. 3 Durchschnittlicher Erholungsflächenbedarf im Kanton Bern (ohne Teil Südjura)

\begin{tabular}{|l|r|r|r|r|r|r|r|r|r|}
\hline \multicolumn{2}{|l|}{ Verteilung der effektiv Erholungsuchenden } \\
auf die Erholungstypen
\end{tabular}

' $60 \%$ der Gesamtzahl, d. h. BECKMANN geht davon aus, daß nur $60 \%$ aller Erholungsuchender gleichzeitig am Ort sind.

${ }^{2}$ Erholungsflächenbedarf in Abhängigkeit des Erholungstyps in ha/P.

${ }^{3}$ Zur Berechnung des Flächenanspruchs der Skifahrer muß davon ausgegangen werden, daß alle gleichzeitig anwesend sind und der mittlere Flächenverbrauch $1 / 20$ ha/Skifahrer beträgt.

nung außer dem Bauern, der das Land verkauft, und dem Bauunternehmer kaum jemandem etwas bringt. Anders verhält es sich dort, wo es gelingt, diese Unterkünfte im Rahmen des von Einheimischen getragenen Tourismus besser auszulasten.

\section{Flächenansprüche des Wochenendtourismus im Raume Bern - ermittelt anhand des Modells von Beckmann (1975)}

Beckmann hat sein Modell mit Hilfe von Fallbeispielen im Ruhrgebiet entwickelt. Voraussetzung für die Anwendung dieses Modells sind die Kenntnis der
Aufgrund der Umfrageergebnisse und der Zahlen einer Verkehrszählung - durchgeführt an zwei der Befragungswochenenden - konnte ein Zupendlerfaktor von 1,07 ermittelt werden, zudem wurde noch der Typ 3 B «Skifahrer» eingeführt.

Interessanterweise ist in diesem Zusammenhang noch darauf hinzuweisen, daß an den winterlichen Schönwettertagen vom 1. und 2.3.1975 aufgrund der entsprechenden Ausflugsintensität (vgl. Tab. 2) eine Gesamtzahl von ca. 56000 Skifahrern am Samstag und 44000 am Sonntag errechnet werden kann. Das ergibt einen Flächenanspruch von ca. 2400 ha, doppelt soviel wie im Durchschnitt. 
Die Berechnung des Erholungsflächenbedarfs für den Schönwettertag vom 2.3. in der Region Oberland-Ost ergab ca. 8500 ha (Typ 1: ca. 3000 ha, Typ 2: ca. 4200 ha, Typ 3A: ca. 100 ha, Typ 3B: ca. 1200 ha). Das regionale Entwicklungskonzept Oberland-Ost (1975) gibt - berechnet aufgrund der verfügbaren Pistenfläche - eine Aufnahmekapazität der Skigebiete von ca. 22000 Skifahrern an. An diesem Samstag wurde aufgrund der Umfrageresultate eine Spitzennachfrage von 23000 Skifahrern errechnet. Ein Blick auf die Jahresganglinie des täglichen Verkehrs auf der $\mathrm{N} 6$ bei Thun zeigt, daß das Angebot der Region 1975 an 8 bis 12 Tagen voll ausgelastet wurde. Die Aufnahmefähigkeit der Transportanlagen betrug 1975 ca. 27500 und 1980 ca. 31000 Skifahrer.

\section{Schlußfolgerungen}

Die Ausflugsintensität der Stadtberner Bevölkerung ist bereits derart groß, daß kaum mit einer wesentlichen Nachfragesteigerung im Wochenendtourismus zu rechnen ist. Bei der weiteren Verbesserung von Verkehrsträgern, insbesondere Autobahnen, kann in einzelnen Fällen eine Vergrößerung der Nachfrage durch das vergrößerte Einzugsgebiet eintreten.

Die große Zahl unterschiedlicher Ausflugsziele und die variantenreiche Freizeitgestaltung verunmöglichen allgemeine Patentlösungen. Die Beseitigung lokaler Engpässe kann im gegebenen Falle ebenso zur Problemlösung beitragen wie die Beibehaltung vorgelagerter Verkehrsengpässe.

Sich ändernde Wertmaßstäbe können zu Trendwendungen führen. «Unberührte Gebiete» müssen dafür bereitstehen, allerdings nur unter Erhaltung ihrer Vorteile. Solche Gebiete sind in Zukunft besonders gefährdet. Ihr Potential ist gering einzuschätzen, weil naturverbundene Freizeittätigkeiten viel größere Flächenansprüche kennen.

Das bestehende Potential kann, abgesehen von einigen wenigen Spitzentagen, der Nachfrage gerecht werden. Der vorhandene Raum läßt auch noch gewissen Spielraum für die Zukunft offen. Unterstützungswürdig sind Bestrebungen zur gleichmäßigeren und daher optimalen Ausnützung des bestehenden Freizeitangebotes. Der einseitige Ausbau einzelner Infrastrukturbereiche (touristische Transportanlagen, Verkehrswege) verhindern optimierte Lösungen. Hingegen können organisatorische Maßnahmen mithelfen, solche Spitzen abzubauen.

Die Resultate dieser Untersuchung weisen darauf hin, daß infrastrukturorientierte Lösungen von vielen Stadtbewohnern unerwünscht sind.

So gesehen, dürfen nicht mehr allein bestehende Verkehrshindernisse, Parkplatzsorgen oder Warteschlangen an Seilbahnen Maßstab zur Beurteilung der Nachfrage sein. Erst wenn es gelingt, mit einer umfassenden Politik, z.B. mit einer zielkonformen
Raumordnungspolitik, die Eigengesetzlichkeiten des Wochenend- und Ferientourismus zu durchbrechen, kann einer schleichenden Zerstörung unseres Lebensraumes wirksam entgegengetreten werden.

Das Ziel, «Erhaltung der Landschaft» zu erreichen, setzt eine Bereitschaft, übliche Entscheidmechanismen beiseite zu lassen, voraus. Für die Freizeit ist dies von eminenter Bedeutung. Es wird deutlich, daß Probleme vielfach auch durch ein inkonsequentes Verhalten des Einzelnen in der Freizeit hervorgerufen werden. Die Diskrepanz zwischen Wunschvorstellungen und Verhalten führten zu «internen Nutzungskonflikten".

Auch die Interessen des Wochenendtourismus und des Ferientourismus brauchen nicht die gleichen zu sein. Obschon z. B. der Bau von Zweitwohnsitzen weiterhin sehr oft mit der Begründung der tourismuspolitischen Notwendigkeit vorangetrieben wird, belegt diese Studie, daß der Zweitwohnungsbau zu einer direkten Konkurrenzierung des Tourismus, insbesondere hinsichtlich seiner regionalwirtschaftlichen Auswirkungen, führen kann. Hotelbetriebe und Gaststätten sind die Arbeitgeber im Tourismus.

\section{Literaturverzeichnis}

ALBRECHT I. (1965): Die Wochenendverkehrsregion Hamburg, Hamburg.

BECKMANN T. (1975): Modell zur Schätzung des Naherholungsflächenbedarfs in Verdichtungsräumen - dargestellt am Beispiel des Ruhrgebietes, Bonn.

BERATENDE KOMMISSION FÜR FREMDENVERKEHR DES BUNDESRATES (1979): Das Schweizerische Tourismuskonzept, Bern.

ELSASSER et al. (1982): Nichttouristische Entwicklungsmöglichkeiten im Berggebiet, Schriftenreihe zur Orts-, Regionalund Landesplanung Nr. 29, Zürich.

FRIEDRICH W. et al. (1979): Naherholung am Beispiel der Region Schwarzenburg, Bern/Thun.

JACSMAN J. (1982): Zur Bestimmung des Flächenbedarfs für die Naherholung. In: DISP Nr. 66, Zürich.

KASPAR C. und STAUB F. (1981): Die Problematik der Zweitwohnungen, Sonderreihe Fremdenverkehr Nr.11, St. Gallen.

KRIPPENDORF J. (1975): Die Landschaftsfresser, Bern/Stuttgart.

REGION OBERLAND-OST (1975): Entwicklungsplanung, Band 1, Lageanalyse, Bern.

SCHWARZENBACH F.H. et al. (1978): Wachstumsprozesse des Skitourismus und Belastung der Landschaft, Hsg.: Eidg. Oberfrontinspektorat, Bern.

SCHMIDHAUSER H.P. (1973): Der Wochenendausflugsverkehr in der Schweiz 1972/73, Hsg.: Stab Gesamtverkehrskonzeption (GVK-CH), St. Gallen und Bern.

THÉLIN G. (1983): Freizeit im Erholungsraum, Arbeitsgemeinschaft Geographica Bernensia, Bern.

VOLKART H.R. (1979): Die Erholungsgebiete im Kanton Zürich, Zürich. 